\title{
Assessment of closely related Mycobacterium tuberculosis variants with different transmission success and in vitro infection dynamics
}

\author{
Estefania Abascal \\ Hospital General Universitario Gregorio Marañón \\ Charlotte Genestet \\ Claude Bernard University Lyon 1 \\ Ana valera \\ Hospital General Universitario Gregorio Marañón \\ Miguel Martinez Lirola \\ Complejo Hospitalario Torrecárdenas \\ Patricia Muñoz \\ Hospital General Universitario Gregorio Marañón \\ Oana Dumitrescu \\ Hospices Civils de Lyon \\ Darío García de Viedma ( $\sim$ dgviedma2@gmail.com ) \\ Hospital General Universitario Gregorio Marañón \\ Marta Herranz \\ Hospital General Universitario Gregorio Marañón
}

\section{Research Article}

Keywords: Tuberculosis, transmission, infectivity

Posted Date: March 10th, 2021

DOI: https://doi.org/10.21203/rs.3.rs-154451/v1

License: @ (i) This work is licensed under a Creative Commons Attribution 4.0 International License. Read Full License 


\section{Abstract}

Whole genome sequencing (WGS) is able to differentiate closely related Mycobacterium tuberculosis variants within the same transmission cluster. Our aim was to evaluate if this higher discriminatory power may help identify and characterize more actively transmitted variants and understand the factors behind their success. We selected a robust MIRU-VNTR-defined cluster from Almería, Spain (22 cases throughout 2003-2019). WGS allowed discriminating, within the same epidemiological setting, between a successfully transmitted variant and seven closely related variants that did not lead to secondary cases, or were involved in self-limiting transmission (one single secondary case). Intramacrophagic growth of representative variants was evaluated in an in vitro infection model using U937 cells. Growth rates were higher for the actively transmitted variant (range: 5.3-10.7) than for the unsuccessfully transmitted closely related variants (1.5-3.95). Two SNPs, mapping at the DNA binding domain of DnaA and at $k d p D$, were found to be specific of the successful variant.

\section{Introduction}

It is estimated that around a quarter of the world's population is infected by Mycobacterium tuberculosis (MTB). Nevertheless, only $5-10 \%$ of the cases progress to active tuberculosis (TB) and are responsible for the transmission to secondary cases. TB can show a wide range of symptoms and clinical severity and the dynamics of its transmission from an infectious case may differ extensively [1].

Historically, it has been assumed that, due to the low genetic diversity within members of the M. tuberculosis complex (MTBC), differences in infection outcomes or dynamics of transmission are linked to the host or environmental factors. However, varying degrees of diversity have been broadly demonstrated for MTBC members, which could also have a potential role in the differences described at clinical or epidemiological levels [2]

To characterize the diversity of MTBC we may tune our analysis, depending on the discriminatory power of the genotyping tools we apply. Lower discriminatory power techniques allow classifying MTB in eight lineages[3-5] that differ not only in their genetic background but also in their geographical distribution[6], transmission efficiency[7, 8], and invasiveness, virulence, etc.[9].

Over the last 25 years, molecular epidemiology approaches have allowed to extend our knowledge on TB transmission and helped improve control measures[10]. The use of infection models allows determining correlations between certain strains that successfully transmit and their ability to replicate within macrophages[11-13].

Whole genome sequencing (WGS) has improved the precision of assessing diversity between MTB isolates, revealing that the standard genotyping tools used until now, including those with greater discriminatory power such as the 24-loci MIRU-VNTR, cannot define clusters accurately[10].

In a population-based MIRU-VNTR systematic analysis including all the TB cases during fourteen consecutive years in the province of Almería (SouthEast Spain), with the majority of TB cases involving migrants, we observed how, within a theoretically robust MIRU-VNTR defined cluster, Cluster 113 (lineage 4.3.2, pansusceptible)[14], it was possible to discriminate by WGS analysis between variants that were successfully transmitted and other closely related variants not responsible of secondary cases or involved in self-limiting small transmission events[14-16]

Cluster 113 is a good example of how WGS allows to accurately reinterpret MIRU-VNTR defined clusters in epidemiologically complex populations with a high rate of migrants $[17,18]$. The most likely interpretation of the topology of the WGS-based network, supported on a strain-specific-PCR-based surveillance in Morocco and the available epidemiological data, is that these variants probably emerged in Morocco after the strain had been long circulating in that country, and were independently exported from Morocco to Almería, Spain[14]. Once exported, certain variant was successfully transmitted in Almería, whereas other did not cause secondary cases

The purpose of this study is to extend the previous efforts based on lower resolution genotyping approaches to the more refined WGS data, to find relationships between diversity and infectivity/transmission efficiency. Based on the subtle differences revealed by WGS between closely related variants within the MIRU-VNTR defined Cluster 113, we will assess whether bacterial factors may have a role in the differential transmission efficiency by coupling WGS analysis with the evaluation of these variants in a macrophage infection model.

\section{Materials And Methods Strain selection}

In a previous study[14], 24-locus-MIRU-VNTR defined clusters from a population-based study carried out in Almería (Spain) (including all the culture-positive TB cases diagnosed in the entire province during 14 consecutive years; 2003-2017), were analysed by WGS, which resulted in the division of closely related isolates ( $\leq 5$ SNPs) and several variants with a higher diversity (> 10 SNPs).

In the present study, we examined the clusters with marked asymmetry as per the WGS analysis; one MTB variant was found in a large number of cases, while other/s were more distantly related variant/s identified in a smaller number of cases. We used the number of cases infected by each variant as proxy of differential transmission success (1-2 cases). These requirements were fulfilled by the complex cluster 113[14].

\section{Macrophage infection model}

We assessed the infectivity and multiplication ability of the selected variants from Cluster 113, represented by successfully transmitted strains or not transmitted (not causing secondary cases) variants, using an in vitro macrophage infection model[19]. 
Briefly (Fig. 1), U937 monocytic cells (monocytic cell line from pleural effusion (ATCC $®$ CRL-1593.2 ${ }^{\text {TM }}$ )) were grown in suspension in RPMI-1640 medium supplemented with $10 \%$ fetal bovine serum (FBS) plus penicillin and streptomycin at final concentrations of $100 \mathrm{U} / \mathrm{ml}$ and $0.1 \mathrm{mg} / \mathrm{ml}$, respectively $(1 \%$ penicillin-streptomycin solution, Sigma-Aldrich, St. Louis, Missouri, USA) to minimize contamination during cell subculture. Monocytes were differentiated to macrophages by adding phorbol 12- myristate 13-acetate at a final concentration of $100 \mathrm{nM}$ and left for 48 hours, following the standard protocol of differentiation of macrophages by PMA stimulation[20]. Following differentiation, the macrophages attached to the bottom of the well in a monolayer were washed once with RPMI media + $10 \%$ foetal bovine serum to remove non-adherent macrophages. After 24 hours, cells were infected, in antibiotic-free medium, with the MTB variants (one single variant tested per well) at a 10:1 (bacteria to cells) multiplicity of infection. For inoculum preparation, MTB variants were grown on egg-based Coletsos medium, collected in exponential phase, at the beginning of colony formation on Coletsos medium, suspended in 3 ml of sterile $0.45 \% \mathrm{NaCl}$ solution $+0.1 \%$ Tween 80 and homogenized by exhaustive vortexing with glass beads in $15 \mathrm{ml}$ glass tubes. The preparation was left for 15 minutes to allow clumps to sediment. Next, homogenized bacteria suspensions were recovered from the upper part and the concentration was adjusted bacterial suspension at $0.5 \mathrm{McF}$, corresponding to approximately $2.10^{7} \mathrm{CFU} / \mathrm{mL}$ according to our lab optimization tests. After four hours of infection, the infected cells were incubated for one hour with amikacin (final concentration of $200 \mu \mathrm{g} / \mathrm{ml}$ ) to kill extracellular and cell-membrane bound bacteria, followed by three washings.

To evaluate invasiveness (Day 0), cells were lysed after the 4-hour incubation period by adding cold water $+0.1 \%$ saponin and scratched thoroughly. Intracellular mycobacteria were recovered and 24-fold 1:2 serial dilutions performed. Next, $7 \mu$ l droplets of each dilution were plated on $7 \mathrm{H} 10$ agar, left for $3-4$ weeks at $37^{\circ} \mathrm{C}$ and then visually inspected to determine colony-forming units (CFUs).

To measure intramacrophage growth amikacin was added at Day 4 post-infection to remove extracellular bacteria and recover intracellular bacteria. Plating and CFU count analysis were performed as described above.

All variants were included in every infection round, to rule out biases due to methodological inter-experiment variability. Additionally, a duplicate infection of every isolate was performed in two different wells at each endpoint to discard intra-experiment deviations (mean results were considered for the statistical analysis). Infections were repeated three times for validation purposes.

\section{Statistical analysis}

Statistical analyses were performed using GraphPad Prism 5. Bacterial counts (Day 0 and Day 4/Day 0 ratio) were expressed as median values \pm interquartile range and compared using the Kruskal-Wallis test followed by Dunn's post-hoc test. A p value $<0.05$ was considered statistically significant.

\section{Analysis of variant specific single-nucleotide polymorphisms}

SNPs were identified from previous WGS data analysis[14]. For all specific SNPs in successfully transmitted variants (present in it but absent in closely related variants), which were coding (calls mapping in genes) and non-synonymous, a review from the literature was performed to determine their potential involvement in MTB virulence/infectivity.

\section{Results And Discussion}

Cluster 113[14] was selected as the source of MTB closely related variants with differential transmission efficacy. We first updated its composition, incorporating WGS data of the new cases identified after its initial description in 2017, in a population-based MIRU-VNTR systematic analysis including all the TB cases in the province during fourteen consecutive years[14]. The updated cluster network (Fig. 1) showed 19 cases, mainly Moroccan migrants (11 cases) and a limited representation of cases from Spain (six cases), Mali (one case), and Nigeria (one case). The network distributed the cases in seven branches, despite sharing identical MIRU-VNTR patterns. The branch with most cases involved Variant 1 (9 cases with $0-1$ SNPs pairwise distances, most likely due to uncontrolled recent transmission). Four branches (involving Variants 2-5) were dead-ends, i.e., single cases not causing secondary cases. The remaining three branches (variants 6-8) represented, self-limiting, short transmission events with only two cases.

We tested the behaviour of two representatives for the actively transmitted Variant 1 (Morocco-2010, index case, and Morocco-2014) in an in vitro macrophage infection model (Fig. 2). Variants 2, 5, and 8-2010 (differing 19-22 SNPs from the successful variant) were selected as non-transmissible controls, which had had the opportunity to be transmitted; the corresponding cases were diagnosed in 2010, 2015 and 2016, respectively, but did not cause any secondary case (Fig. 2).

We first assessed dissimilarities in invasiveness. No statistical differences were found between Variant 1 and the non-transmitted control variants (Fig. 3a).

Next, we evaluated their intramacrophage multiplication rates. We observed a statistically significant superiority of Variant 1 based on intracellular growth (Fig. 3b). The multiplication ratios for Variant 1 were 2-4 folds higher than the obtained for the non-transmitted variants (ratios ranging from 5.3 to 10.7 vs from 1.5 to 3.95 , respectively; $p=0.0244$ ).

Certain outbreak strains, namely CDC1551, demonstrated high transmissibility but were not found to be more virulent[21, 22]. For Cluster 113, the higher intramacrophage multiplication observed for Variant 1 led us to a further detailed analysis to determine if the differential SNPs found between this variant and the control ones could explain that behaviour. Among the nine specific SNPs identified exclusively in Variant 1 (Table 1), seven were homozygous calls for all isolates, but the remaining two were heterozygous in the index case and were thus fixed in the rest. Two SNPs were located in intergenic regions, which could play a role, if they relate to regulatory elements. For those intragenic, we focused on the (four) corresponding to non-synonymous mutations. Two of them mapped on genes without a described function (the Rv0090 and Rv1517 hypothetical proteins), while the other two may have a functional meaning (Rv0001, alias $d n a A$, and Rv1028c, alias $k d p D)$. 
Table 1

Specific SNP annotation for the successfully transmitted Cluster 113 variant

\begin{tabular}{|c|c|c|c|c|c|c|c|c|c|c|c|}
\hline $\begin{array}{l}\text { SNP } \\
\text { position }\end{array}$ & $\begin{array}{l}\text { Nucleotide } \\
\text { change }\end{array}$ & $\begin{array}{l}\text { Allele } \\
\text { freq } \\
\text { index* }\end{array}$ & Gene & \multicolumn{2}{|c|}{ Gene coordinates } & $\begin{array}{l}\text { Gene } \\
\text { direction }\end{array}$ & \multicolumn{2}{|l|}{$\begin{array}{l}\text { Essential } \\
\text { character }\end{array}$} & \multirow{2}{*}{$\begin{array}{l}\text { Protein } \\
\text { length }\end{array}$} & \multicolumn{2}{|c|}{ Aminoacid change } \\
\hline 1163 & $\mathrm{C}>\mathrm{T}$ & 0.29 & $\begin{array}{l}\text { Rv0001 } \\
\text { (dnaA) }\end{array}$ & $\begin{array}{l}1 \ldots \\
1524\end{array}$ & + & esser & & 507 & & $\mathrm{~T}>\mathrm{I}$ & chromosomal repl \\
\hline 1623635 & $G>A$ & 0.5 & Rv1444c & $\begin{array}{c}1623287 \ldots \\
1623697\end{array}$ & - & non-e & sential & 136 & & Synonymous & hypothetical prote \\
\hline 99141 & $\mathrm{~T}>\mathrm{G}$ & $>0.9$ & Rv0090 & $\begin{array}{l}98480 \ldots \\
99250\end{array}$ & + & non-e & sential & 256 & & $L>R$ & hypothetical prote \\
\hline 1151418 & $C>T$ & $>0.9$ & $\begin{array}{l}\text { Rv1028c } \\
(k d p D)\end{array}$ & $\begin{array}{c}1149104 \ldots \\
1151686\end{array}$ & - & non-e & sential & 860 & & $\mathrm{R}>\mathrm{Q}$ & sensor protein $\mathrm{Kdr}$ \\
\hline 1163957 & $C>T$ & $>0.9$ & $\begin{array}{l}\text { IG1057 } \\
\text { (Rv1040c- } \\
\text { Rv1041c) }\end{array}$ & $\begin{array}{l}1163377 \ldots \\
1164571\end{array}$ & & & & & & & \\
\hline 1709423 & $\mathrm{G}>\mathrm{T}$ & $>0.9$ & Rv1517 & $\begin{array}{l}1708871 \ldots \\
709635\end{array}$ & + & non-e & sential & 254 & & $\mathrm{G}>\mathrm{C}$ & hypothetical prote \\
\hline 2142832 & $C>T$ & $>0.9$ & Rv1895 & $\begin{array}{l}2142521 \ldots \\
2143675\end{array}$ & + & non-e & sential & 384 & & Synonymous & possible dehydrog \\
\hline 3659433 & $G>A$ & $>0.9$ & $\begin{array}{l}\text { Rv3276c } \\
\text { (purK) }\end{array}$ & $\begin{array}{l}3658635 \ldots \\
3659924\end{array}$ & - & non-e & sential & 429 & & Synonymous & $\begin{array}{l}\text { phosphoribosylan } \\
\text { carboxylase ATPa: }\end{array}$ \\
\hline 4314130 & $\mathrm{~T}>\mathrm{C}$ & $>0.9$ & $\begin{array}{l}\text { IG3905 } \\
\text { (Rv3840- } \\
\text { Rv3841) }\end{array}$ & $\begin{array}{l}4313981 \ldots \\
4314177\end{array}$ & & & & & & & \\
\hline
\end{tabular}

* Allele frequency in all secondary cases was $>0.9$

$d n a A$ encodes a key protein for triggering the chromosomal replication machinery at oriC through interactions with the DnaA-boxes, which assembles a nucleoprotein complex responsible for the ATP-dependent opening of the double strand of DNA[23]. The differential SNP found between this variant and the control ones maps at residue 388 of the DNA binding domain of DnaA[24] and causes a threonine (polar uncharged amino acid) to isoleucine (hydrophobic amino acid) substitution. Although modelling data are required to interprete the meaning of this substitution, we might speculate that the nature of this substitution may improve DnaA-oriC interactions. The fact that the SNP mapping in dnaA was initially detected in heterozygosis in the index case and was then fixed in all secondary cases, may reinforce the advantageous character of this substitution.

$k d p D$ is one of the two genes that constitute the $k d p D E$ operon, one of the scarce two-component regulatory systems (2CRSs) found in MTB, and it encodes its sensor protein[25]. It has been shown that strains with deletions in $k d p D E$, and in some other MTB 2CRSs, increase their virulence in an immunodeficient mouse model[26]. Folkvardsen et al.[15] identified a specific SNP in a 2CRS from a strain responsible for one of the major transmission events worldwide currently active- not present in a closely related variant that caused few secondary cases. This SNP is located in the tcrY[15] gene, which also codes for the sensor TcrXY protein of a 2 CRS system. It has also been proven that deletions in this 2 CRS system results in a hypervirulent phenotype in SCID mice[26]. We must admit that several of the observations related to the virulence of mutants in 2CRS systems were obtained from immunocompromised models and might not be transferable to an inmunocomponent system, such as the cases from which Variant 1 was obtained.

The KdpDE 2CRS system is involved in the detection and response of $\mathrm{pH}$ and $\mathrm{K}+$ changes (related with turgor pressure, regulation of cytoplasmic $\mathrm{pH}$, osmolarity, transmembrane electrical potential, etc.) by modulating the expression of a $\mathrm{K}+$ transport system[27]. This system may have a role in MTB efficiency to survive acidic environments, e.g., phagosome or autophagosome vacuoles[27]. The specific SNP harboured by Variant 1 at the N-terminal domain of this protein implies an arginine (positively charged amino acid) to glutamine (polar uncharged amino acid) substitution. The domain interacts with two lipoproteins, LprF and LprJ, to form a ternary complex that modulates the sensing ability of KdpD[28]. Thus, the substitution described for Variant 1 may affect the binding affinity of Lpr and the subsequent expression of the $\mathrm{K}+$ transport system, and consequently the modulation of pH and $\mathrm{K}+$ uptake. However, these are mere hypothesis that require further validation/evidence.

In summary, we have identified two specific SNPs in Variant 1, located in $d n a A$ and $k d p D$, not found in other closely related non-transmitted variants. The changes caused by these SNPs may be associated to the higher infectivity shown by Variant 1 in macrophages. We cannot fully exclude the role of other potentially undetected mutations. This is because repetitive regions, including, among others, the PE and PPE genes, must still be systematically excluded from any Illumina-based WGS analysis. It causes the existence of black boxes with unknown diversity[29], in regions known to be essential for MTB virulence and interaction with the host immune system[30]. 
In addition to environmental and host-related factors, our study suggests that bacterial factors must also be considered when aiming to understand major transmission events. WGS analysis coupled with a simple in vitro infection model may provide a rapid screening platform of closely related variants with a differential transmission success. Once the involvement of bacterial factors is shown, additional studies on the candidate genes and polymorphisms should be required to more thoroughly characterize them and finally define determinants of MTB virulence, transmissibility, or evasion of the immune system. Targeting these SNPs with tailored allele-specific PCRs may allow fast tracking of strains requiring special surveillance.

\section{Declarations}

\section{ACKNOWLEDGMENTS}

This work was supported by ERANet-LAC (ELAC2015/T08-0664) and Instituto de Salud Carlos III (AC16/00057, FIS15/01554, FIS 18/00599) cofounded by European Regional Development Funds from the European Commission: "A way of making Europe". EA was holder of a grant from CIBERES (Ayudas de perfeccionamiento y movilidad; G9080057).

The authors are grateful to Dainora Jaloveckas (cienciatraducida.com) for editing and proofreading assistance.

\section{The authors do not have commercial or other associations that might pose a conflict of interest}

\section{References}

1. Coscolla M, Gagneux S. Consequences of genomic diversity in Mycobacterium tuberculosis. Semin Immunol 2014; 26:431-44.

2. Gagneux S. Ecology and evolution of Mycobacterium tuberculosis. Nat Rev Microbiol 2018; 16:202-13.

3. Firdessa R, Berg S, Hailu E, et al. Mycobacterial lineages causing pulmonary and extrapulmonary tuberculosis, Ethiopia. Emerg Infect Dis 2013; 19:460-3.

4. Gagneux S, DeRiemer K, Van T, et al. Variable host-pathogen compatibility in Mycobacterium tuberculosis. Proc Natl Acad Sci U S A 2006; 103:2869-73.

5. Ngabonziza JCS, Loiseau C, Marceau M, et al. A sister lineage of the Mycobacterium tuberculosis complex discovered in the African Great Lakes region. Nat Commun 2020; 11:2917.

6. Stucki D, Brites D, Jeljeli L, et al. Mycobacterium tuberculosis lineage 4 comprises globally distributed and geographically restricted sublineages. Nat Genet 2016; 48:1535-43.

7. Asare P, Asante-Poku A, Prah DA, et al. Reduced transmission of Mycobacterium africanum compared to Mycobacterium tuberculosis in urban West Africa. Int J Infect Dis 2018; 73:30-42.

8. Holt KE, McAdam P, Thai PVK, et al. Frequent transmission of the Mycobacterium tuberculosis Beijing lineage and positive selection for the EsxW Beijing variant in Vietnam. Nat Genet 2018; 50:849-56.

9. Lopez B, Aguilar D, Orozco H, et al. A marked difference in pathogenesis and immune response induced by different Mycobacterium tuberculosis genotypes. Clin Exp Immunol 2003; 133:30-7.

10. Garcia De Viedma D, Perez-Lago L. The evolution of genotyping strategies to detect, analyze, and control transmission of tuberculosis. Microbiol Spectr 2018; 6.

11. Yokobori N, Monteserin J, Rearte B, et al. Survival of an epidemic MDR strain of Mycobacterium tuberculosis and its non-prosperous variant within activated macrophages. Infect Genet Evol 2019; 73:248-54.

12. Theus SA, Cave MD, Eisenach $\mathrm{K}$, et al. Differences in the growth of paired Ugandan isolates of Mycobacterium tuberculosis within human mononuclear phagocytes correlate with epidemiological evidence of strain virulence. Infect Immun 2006; 74:6865-76.

13. Theus SA, Cave MD, Eisenach KD. Intracellular macrophage growth rates and cytokine profiles of Mycobacterium tuberculosis strains with different transmission dynamics. J Infect Dis 2005; 191:453-60.

14. Abascal E, Perez-Lago L, Martinez-Lirola M, et al. Whole genome sequencing-based analysis of tuberculosis (TB) in migrants: rapid tools for cross-border surveillance and to distinguish between recent transmission in the host country and new importations. Euro Surveill $2019 ; 24$.

15. Folkvardsen DB, Norman A, Andersen AB, Michael Rasmussen E, Jelsbak L, Lillebaek T. Genomic epidemiology of a major Mycobacterium tuberculosis outbreak: retrospective cohort study in a low-incidence setting using sparse time-series sampling. J Infect Dis 2017; 216:366-74.

16. Bigi MM, Lopez B, Blanco FC, et al. Single nucleotide polymorphisms may explain the contrasting phenotypes of two variants of a multidrug-resistant Mycobacterium tuberculosis strain. Tuberculosis (Edinb) 2017; 103:28-36.

17. Stucki D, Ballif M, Egger M, et al. Standard genotyping overestimates transmission of Mycobacterium tuberculosis among immigrants in a low-incidence country. J Clin Microbiol 2016; 54:1862-70.

18. Walker TM, Lalor MK, Broda A, et al. Assessment of Mycobacterium tuberculosis transmission in Oxfordshire, UK, 2007-12, with whole pathogen genome sequences: an observational study. Lancet Respir Med 2014; 2:285-92.

19. Genestet C, Bernard-Barret F, Hodille E, et al. Antituberculous drugs modulate bacterial phagolysosome avoidance and autophagy in Mycobacterium tuberculosisinfected macrophages. Tuberculosis (Edinb) 2018; 111:67-70.

20. Chanput W, Peters V, Wichers H. THP-1 and U937 Cells. In: Verhoeckx K, Cotter P, López-Expósito I, Kleiveland C, Lea T, Mackie A, eds. The impact of food bioactives on health. Springer, Cham, 2015:147-59.

21. Bishai WR, Dannenberg AM, Jr., Parrish N, et al. Virulence of Mycobacterium tuberculosis CDC1551 and H37Rv in rabbits evaluated by Lurie's pulmonary tubercle count method. Infect Immun 1999; 67:4931-4. 
22. Manca C, Tsenova L, Barry CE, 3rd, et al. Mycobacterium tuberculosis CDC1551 induces a more vigorous host response in vivo and in vitro, but is not more virulent than other clinical isolates. J Immunol 1999; 162:6740-6.

23. Chauhan S, Tyagi JS. Analysis of transcription at the oriC locus in Mycobacterium tuberculosis. Microbiol Res 2011; 166:508-14.

24. Xie Y, He ZG. Characterization of physical interaction between replication initiator protein DnaA and replicative helicase from Mycobacterium tuberculosis H37Rv. Biochemistry (Mosc) 2009; 74:1320-7.

25. Parish T. Two-component regulatory systems of mycobacteria. Microbiol Spectr 2014; 2:MGM2-0010-2013.

26. Parish T, Smith DA, Kendall S, Casali N, Bancroft GJ, Stoker NG. Deletion of two-component regulatory systems increases the virulence of Mycobacterium tuberculosis. Infect Immun 2003; 71:1134-40.

27. Cholo MC, van Rensburg EJ, Osman AG, Anderson R. Expression of the genes encoding the Trk and Kdp potassium transport systems of Mycobacterium tuberculosis during growth in vitro. Biomed Res Int 2015; 2015:608682.

28. Steyn AJ, Joseph J, Bloom BR. Interaction of the sensor module of Mycobacterium tuberculosis H37Rv KdpD with members of the Lpr family. Mol Microbiol 2003; 47:1075-89.

29. Phelan JE, Coll F, Bergval I, et al. Recombination in PE/PPE genes contributes to genetic variation in Mycobacterium tuberculosis lineages. BMC Genomics 2016; 17:151.

30. Delogu G, Brennan MJ, Manganelli R. PE and PPE genes: a tale of conservation and diversity. Adv Exp Med Biol 2017; 1019:191-207.

\section{Table}

Table 1. Specific SNP annotation for the successfully transmitted Cluster 113 variant

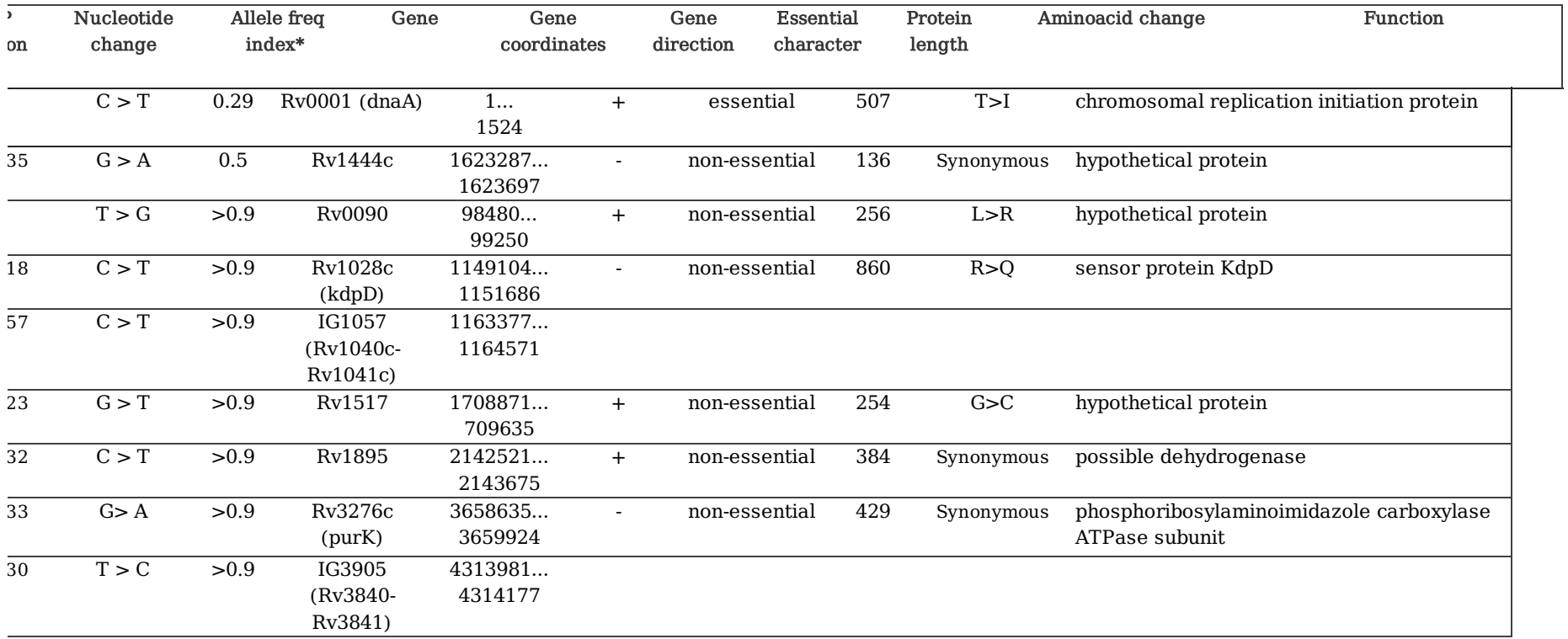

* Allele frequency in all secondary cases was $>0.9$

\section{Figures}




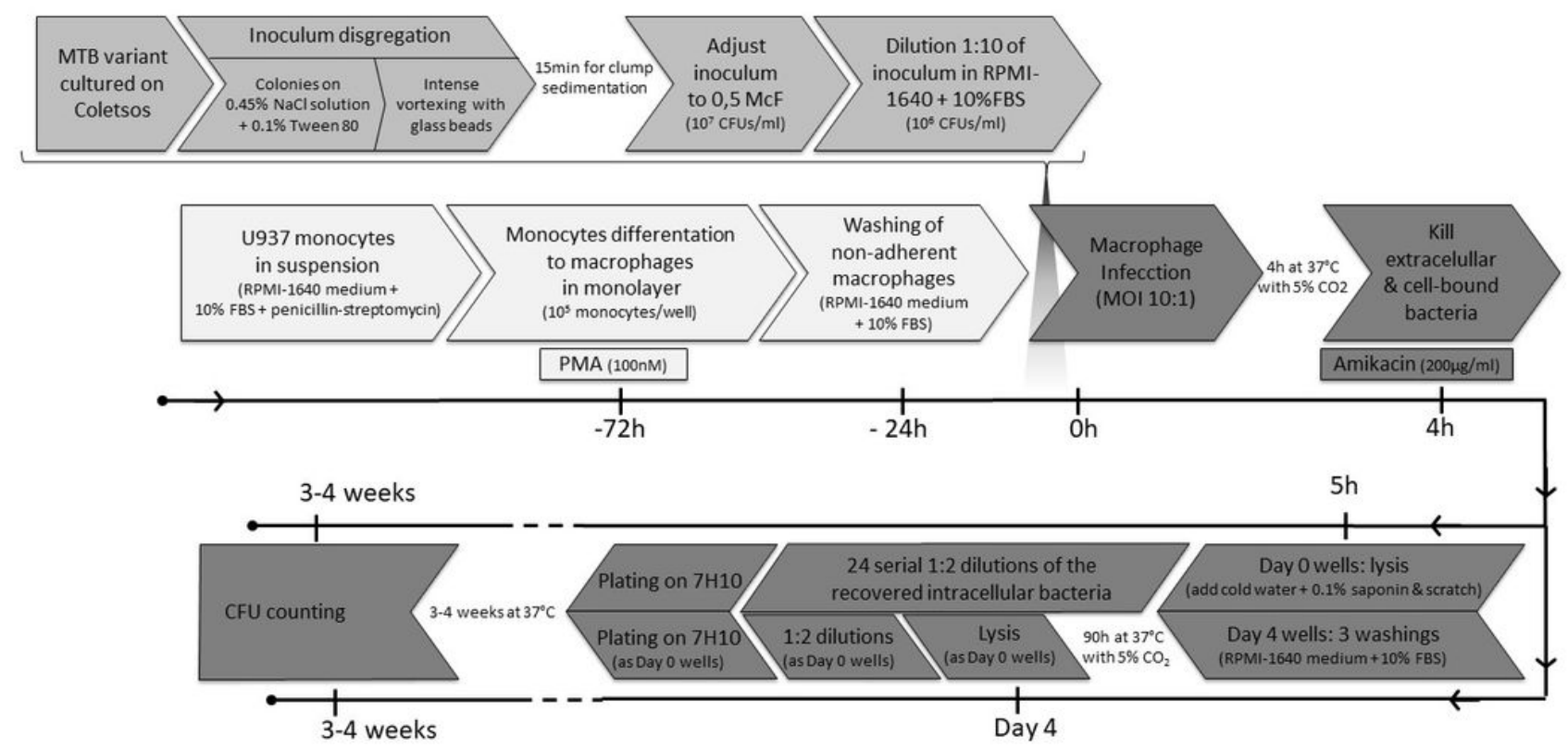

\section{Figure 1}

Flow chart indicating the steps in the infection model.

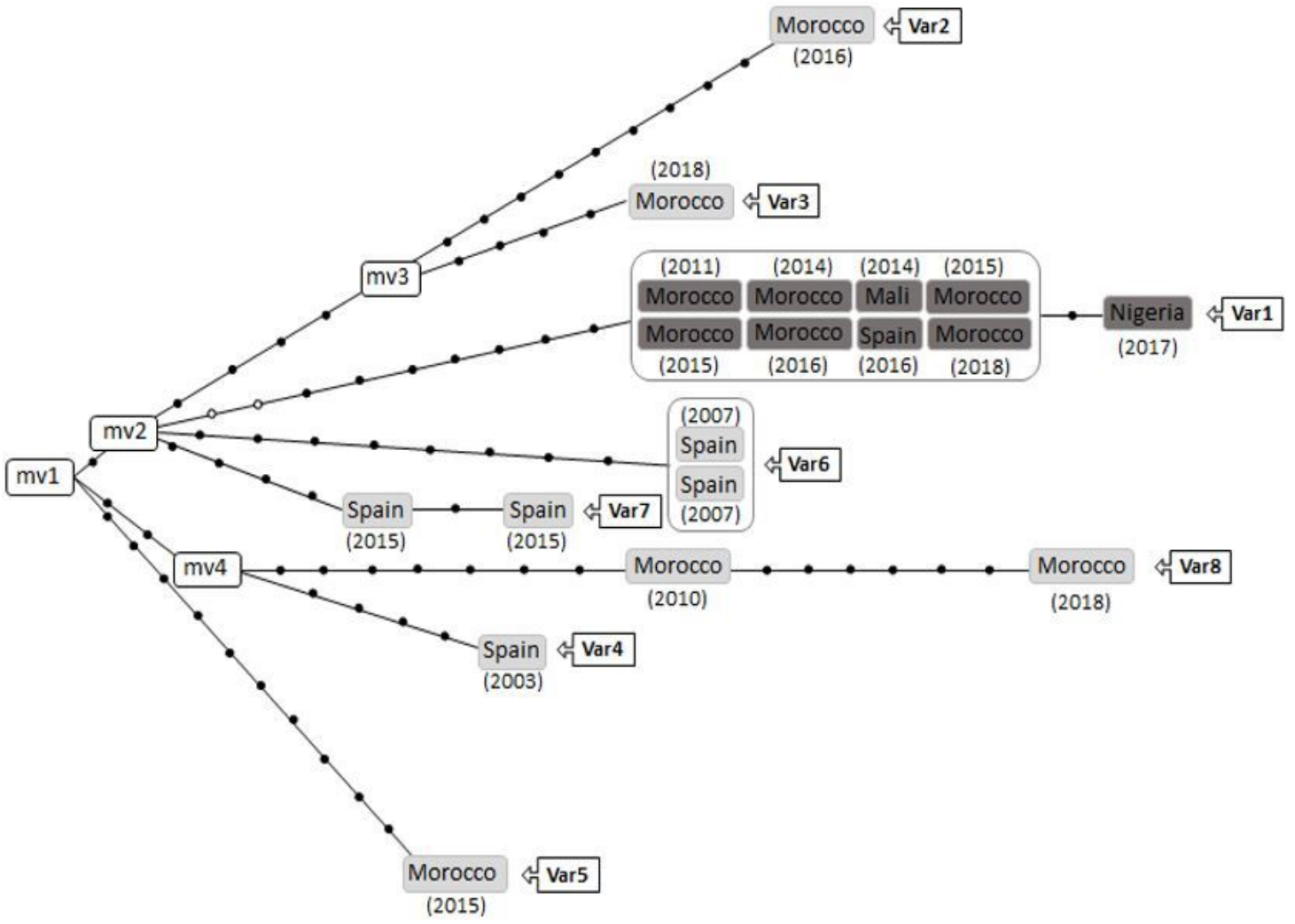

Figure 2

Each box represents a patient, differentiating the ones infected by the successfully transmitted variant (Variant 1, coloured in dark grey) from those infected by unsuccessfully transmitted closely related variants (in light grey). Patient's origin is indicated within the box and the year of diagnosis in brackets. When two or more cases share identical sequences (0 SNPs between them) they are boxed, surrounded by a line. Each black dot represents a SNP. The white circles in 
Variant 1 branch correspond to non-fixed alleles, found in heterozygosis in the index case (Morocco-2011) but in homozygosis in the remaining cases. In the white boxes the name of the variant is indicated (Var). mv: median vectors (corresponding to not-sampled nodes)

Figure $3 a$

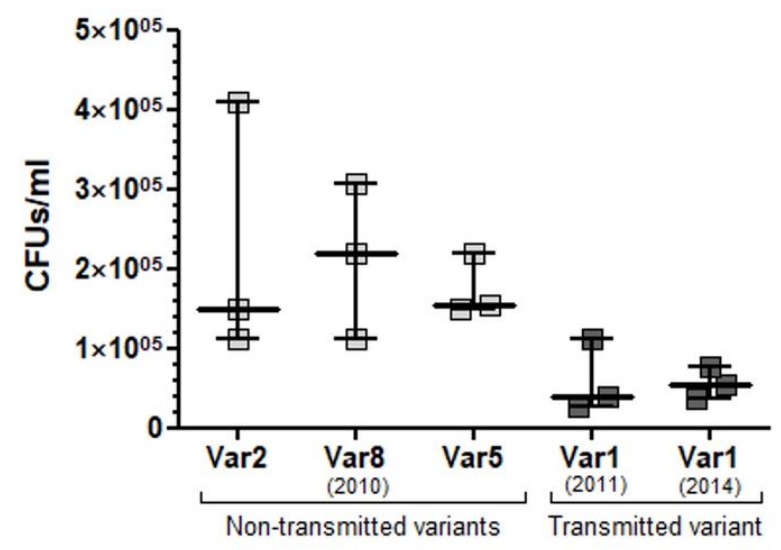

Figure $3 b$

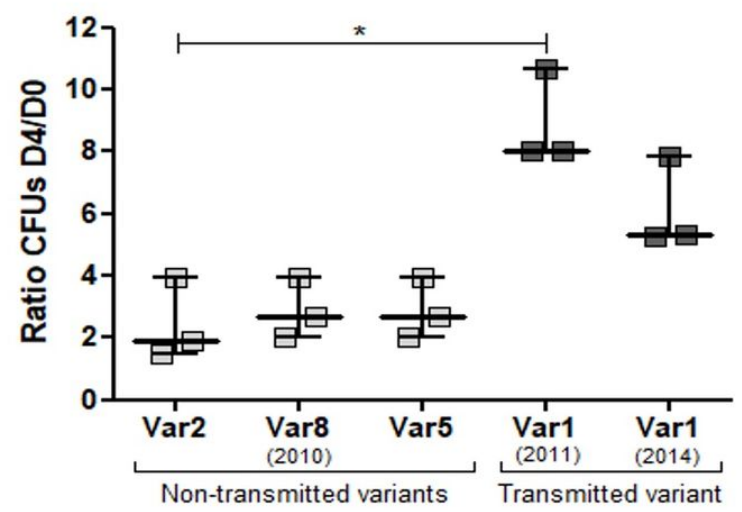

Figure 3

a) Macrophage invasiveness (CFUs at Day 0) for the five selected variants. Each square represents the mean value of an experiment. Lines and error bars indicate median values \pm range. b) Intramacrophage multiplication ratios (CFUs at Day 4 / CFUs at Day 0) for the five selected variants. Each square represents the mean value of an experiment. Lines and error bars indicate median values \pm interquartile range. * $p<0.05$ 